OPEN ACCESS

Edited by:

Keshav Raj Paudel, University of Technology

Sydney, Australia

Reviewed by:

Carolina Vitale

University of Salerno, Italy

Alvaro Teijeiro,

Pediatric Hospital of

Cordoba, Argentina

*Correspondence:

Jeng-Yuan Hsu

hsujy@vghtc.gov.tw

tThese authors have contributed equally to this work and share senior authorship

Specialty section:

This article was submitted to

Pulmonary Medicine,

a section of the journal

Frontiers in Medicine

Received: 21 August 2021 Accepted: 01 December 2021 Published: 03 January 2022

Citation:

Huang W-C, Fu P-K, Chan M-C,

Chin C-S, Huang W-N, Lai K-L,

Wang J-L, Hung W-T, Wu Y-D, Hsieh C-W, Wu M-F, Chen Y-H and Hsu J-Y (2022) Predictors of a Minimal

Clinically Important Difference Following Omalizumab Treatment in Adult Patients With Severe Allergic

Asthma. Front. Med. 8:762318.

doi: 10.3389/fmed.2021.762318

\section{Predictors of a Minimal Clinically Important Difference Following Omalizumab Treatment in Adult Patients With Severe Allergic Asthma}

\author{
Wei-Chang Huang 1,2,3,4,5, , Pin-Kuei Fu, $7,8,9$, Ming-Cheng Chan ${ }^{2,10}$, Chun-Shih Chin ${ }^{1}$, \\ Wen-Nan Huang ${ }^{2,11}$, Kuo-Lung Lai ${ }^{11}$, Jiun-Long Wang ${ }^{1,3,12,13}$, Wei-Ting Hung ${ }^{11}$, Yi-Da Wu ${ }^{11}$, \\ Chia-Wei Hsieh ${ }^{3,11}$, Ming-Feng Wu ${ }^{1,14}$, Yi-Hsing Chen ${ }^{11,15 \dagger}$ and Jeng-Yuan Hsu ${ }^{16,17 * t}$ \\ 1 Division of Chest Medicine, Department of Internal Medicine, Taichung Veterans General Hospital, Taichung, Taiwan, \\ ${ }^{2}$ College of Medicine, National Chung Hsing University, Taichung, Taiwan, ${ }^{3}$ Ph.D. Program in Translational Medicine, National \\ Chung Hsing University, Taichung, Taiwan, ${ }^{4}$ School of Medicine, Chung Shan Medical University, Taichung, Taiwan, ${ }^{5}$ Master \\ Program for Health Administration, Department of Industrial Engineering and Enterprise Information, Tunghai University, \\ Taichung, Taiwan, ${ }^{6}$ Department of Medical Technology, Jen-Teh Junior College of Medicine, Nursing and Management, \\ Miaoli, Taiwan, ${ }^{7}$ Department of Critical Care Medicine, Taichung Veterans General Hospital, Taichung, Taiwan, ${ }^{8}$ College of \\ Human Science and Social Innovation, Hungkuang University, Taichung, Taiwan, ${ }^{9}$ Department of Computer Science, Tunghai \\ University, Taichung, Taiwan, ${ }^{10}$ Division of Critical Care and Respiratory Therapy, Department of Internal Medicine, Taichung \\ Veterans General Hospital, Taichung, Taiwan, ${ }^{11}$ Division of Allergy, Immunology and Rheumatology, Department of Internal \\ Medicine, Taichung Veterans General Hospital, Taichung, Taiwan, ${ }^{12}$ Agricultural Biotechnology Research Center, National \\ Chung Hsing University, Taichung, Taiwan, ${ }^{13}$ Department of Life Sciences, National Chung Hsing University, Taichung, \\ Taiwan, ${ }^{14}$ Department of Medical Laboratory Science and Biotechnology, Central Taiwan University of Science and \\ Technology, Taichung, Taiwan, ${ }^{15}$ School of Medicine, National Yang Ming Chia Tung University, Taipei, Taiwan, ${ }^{16}$ Division of \\ Clinical Research, Department of Medical Research, Taichung Veterans General Hospital, Taichung, Taiwan, ${ }^{17}$ School of \\ Physical Therapy, Chung-Shan Medical University, Taichung, Taiwan
}

Several factors have been found to be predictors of a good response following omalizumab treatment in patients with severe allergic asthma (SAA). However, it remains unclear whether clinical characteristics can predict a minimal clinically important difference (MCID) following omalizumab treatment in this population. Therefore, the aim of this study was to investigate the features associated with an MCID following omalizumab treatment in adult patients with SAA. Of the 124 participants enrolled in this retrospective, cross-sectional study, 94, 103, 20 and 53 achieved the MCID following treatment with omalizumab and were considered to be responders of exacerbation reduction (no exacerbation during the 1 -year follow-up period or $\geqq 50 \%$ reduction in exacerbations from baseline), oral corticosteroid (OCS) sparing (no use of OCS to control asthma during the study period or a reduction of the monthly OCS maintenance dose to $<50 \%$ of baseline), lung function (an increase of $\geqq 230 \mathrm{ml}$ in the forced expiratory volume in $1 \mathrm{~s}$ from baseline) and asthma control (an increase of $\geqq 3$ points in the asthma control test score from baseline), respectively. Normal weight $\left[<25 \mathrm{vs}\right.$. $\geqq 30 \mathrm{~kg} / \mathrm{m}^{2}$, odds ratio $(\mathrm{OR})=3.86, p=0.024$ ] was predictive of a responder of reduction in exacerbations following omalizumab treatment while subjects with a blood eosinophil level of 


\begin{abstract}
$<300$ cells $/ \mu \mathrm{L}(<300$ vs. $\geqq 300$ cells $/ \mu \mathrm{L}, \mathrm{OR}=5.81, p=0.001)$ were more likely to exhibit an MCID in OCS sparing. No factor was found to be a predictor of lung function or asthma control. When choosing treatment for adult patients with SAA, our findings may help to select those who may benefit the most from omalizumab treatment.
\end{abstract}

Keywords: anti-IgE, asthma, minimal clinically important difference (MCID), omalizumab, predictor

\section{INTRODUCTION}

Asthma is a heterogeneous respiratory disease that involves airflow limitation due to chronic airway inflammation. It has been reported to affect $1-18 \%$ of the general population and is categorized into five Global Initiative for Asthma (GINA) steps (Step 1-5) based on the strength of treatment needed to control symptoms and exacerbations. Severe asthma, defined as asthma which cannot be controlled despite the use of GINA Step 4 or 5 pharmacological therapies, good inhaler technique and adherence, and optimal management of contributory factors, is estimated to occur in $3.6 \%$ of asthmatic patients $(1,2)$.

Omalizumab is an anti-immunoglobulin E ( IgE) monoclonal antibody that reduces circulatory free IgE, and it is approved for the treatment of moderate to severe IgE-mediated asthma. The GINA recommendations suggest the use of omalizumab as add-on therapy for severe asthma owing to its noticeable impact on medical resource utilization, quality of life and beneficial treatment outcomes, including improved exacerbation rate, reduced oral corticosteroid (OCS) maintenance dose, better asthma control and improvements in lung function in both adult and pediatric patients with severe allergic asthma (SAA) $(1,3-10)$.

The statistical significance is the most widely used evidence to guide treatment decision making in both clinical trials and daily practice while this does not necessarily imply the clinical relevance. To overcome this gap, it is crucial to determine the minimal clinically important difference (MCID), first described in 1989 by Jaeschke et al. and defined as the smallest improvement in a treatment outcome considered worthwhile by an individual patient, for healthcare providers. Several MCID cut-off values have been proposed and validated in the population of asthma, with most of the cut-offs are associated with patientreported outcomes, lung function and exercise tolerance (11-14). Nevertheless, the MCID has rarely used as a tool for assessing the treatment response of biologics for patients with severe asthma.

The GINA guidelines state that a blood eosinophil count $\geqq 260$ cells/ $\mu \mathrm{L}$, a fractional exhaled nitric oxide (FeNO) level $\geqq 20$ parts per billion, the presence of allergen-driven symptoms, and childhood-onset asthma are statistically significant predictors for a good therapeutic response to omalizumab in reducing exacerbations for patients with SAA (1). However, little is known about whether clinical characteristics are associated with the MCIDs, particularly those regarding the goals of asthma management proposed by the GINA strategy (1), following treatment with omalizumab in this population.

We hypothesized that the baseline clinical features could predict a worthwhile response to omalizumab as an add-on therapy for patients with SAA. Therefore, we investigated the pre-omalizumab treatment clinical characteristics associated with an MCID in reducing exacerbations, OCS sparing, and improvements in lung function and asthma control, the most representative and clinically vital treatment goals recommended by the GINA strategy (1), following treatment with omalizumab in adult patients with SAA.

\section{MATERIALS AND METHODS}

\section{Study Design, Setting, and Population}

This retrospective cross-sectional study was approved by the Institutional Review Board and Ethics Committee of Taichung Veterans General Hospital (TCVGH) (Approval No. CE19015B) and implemented in accordance with the Declaration of Helsinki. The need for informed consent from participants was waived because of the retrospective nature of this study and data extraction based on an electronic medical chart review. The study was conducted at TCVGH, a tertiary referral center in central Taiwan, be-tween January 2010 and January 2019, and enrolled patients diagnosed of SAA who applied for reimbursements for omalizumab from the Taiwan National Health Insurance (NHI) according to the judgment of very experienced pulmonologists and immunologists in charge of asthma management. Patients whose applications were not ap-proved were excluded from this study (8).

\section{Data Collection}

As detailed elsewhere (8), the investigators collected clinical data, including base-line demographics, clinical features and laboratory findings, medications related to asthma management, and co-morbidities for each participant. Moreover, the treatment outcomes of interest, including exacerbation history, usage of OCSs, spirometric data and asthma control test (ACT) scores were also recorded at baseline and the end of 1-year follow-up (8).

\section{Definition of MCID (Responder) According to Treatment Outcome of Interest}

Responders with regards to a reduction in exacerbations were defined as those who had no exacerbations during the study period or who had a $\geqq 50 \%$ reduction in the number of exacerbations in the 1-year follow-up period compared to the year prior to enrollment. An exacerbation was defined as a worsening of respiratory symptoms and lung function that required OCS treatment for $\geqq 3$ days at an outpatient service, emergency visit or hospitalization $(15,16)$. The patients who did not meet these criteria were defined as non-responders. 
Responders with regards to OCS sparing were defined as those who did not use OCS to control asthma during the study period, or whose monthly OCS maintenance dose at the end of study was $<50 \%$ compared to that at enrollment. Maintenance pharmacological therapy for SAA was defined as $>7$ days of OCS prescriptions in the outpatient department. The patients who did not meet these criteria were defined as non-responders.
The patients with an MCID according to lung function and ACT following omalizumab treatment were defined as those with an increase of $\geqq 230 \mathrm{ml}$ and $\geqq 3$ points in the forced expiratory volume in $1 \mathrm{~s}$ (FEV1) and ACT score, respectively, between the end and start of the 1-year follow-up period (13, 14). The patients who did not meet these criteria were defined as non-responders.

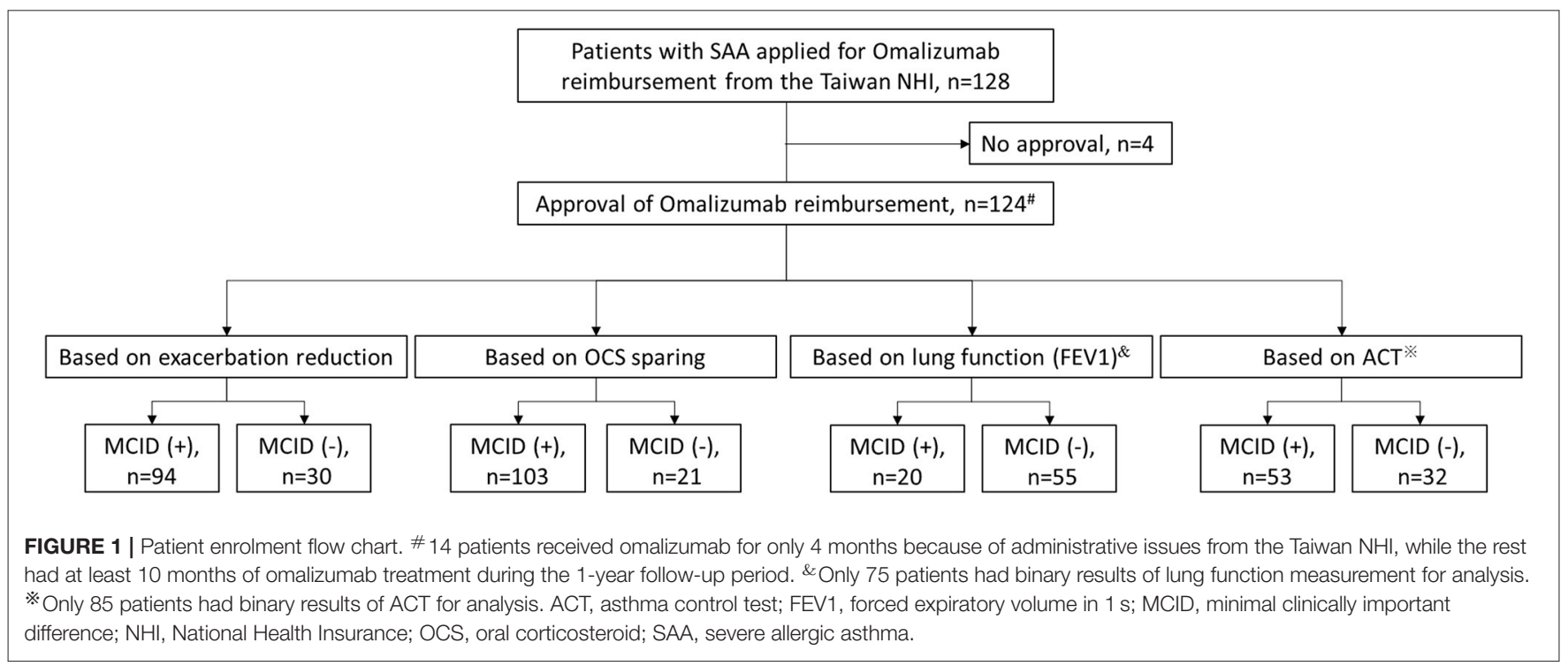

A

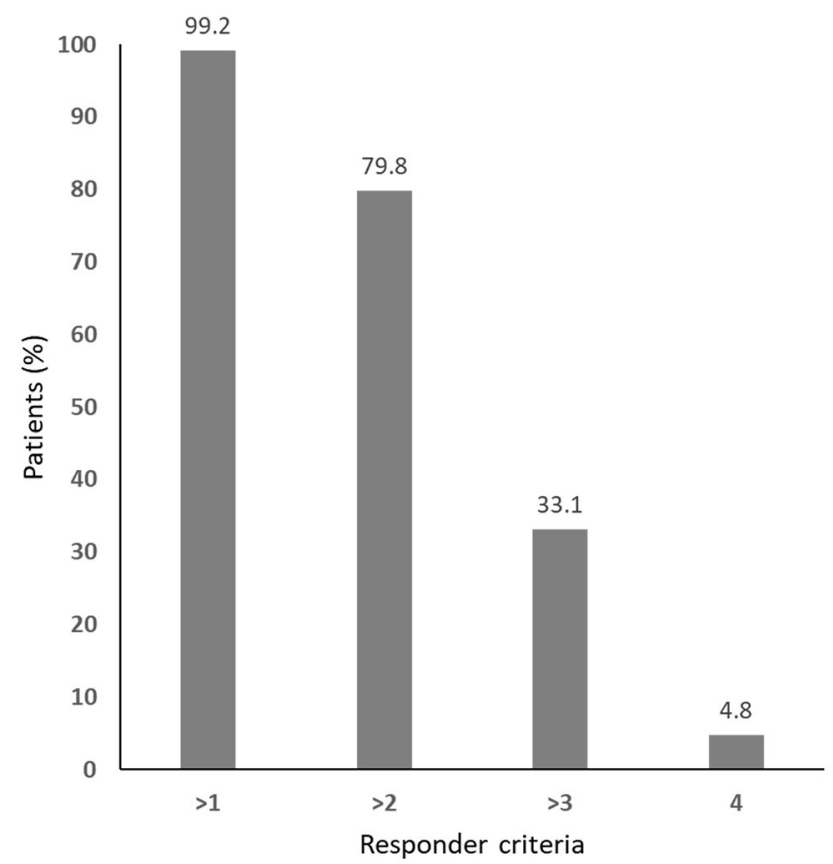

B

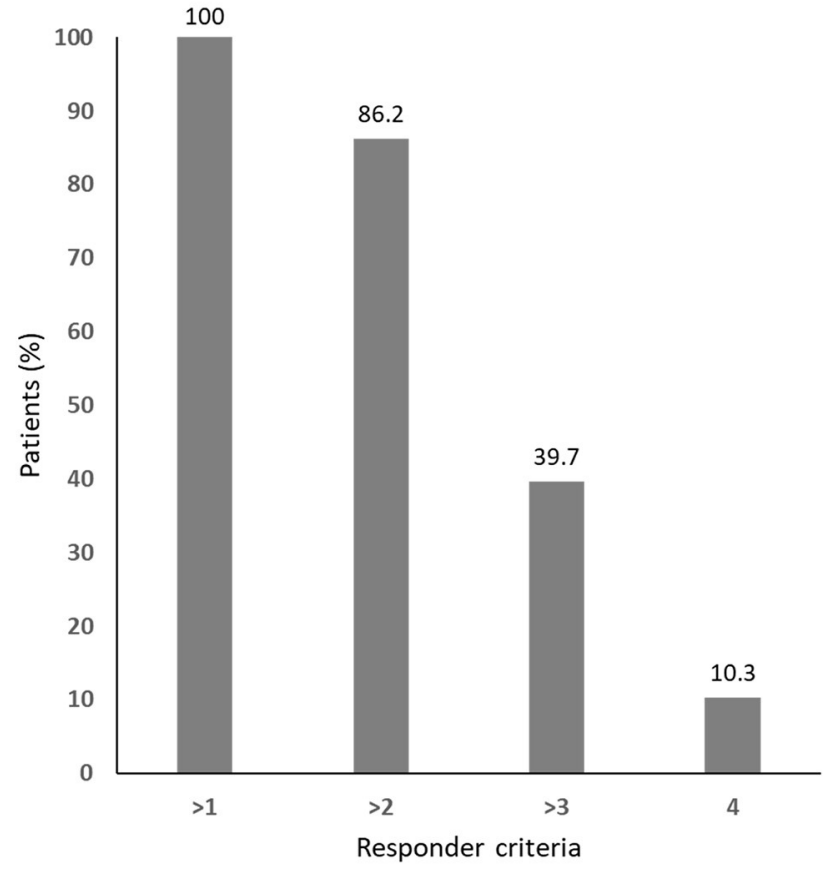

FIGURE 2 | The responder distribution for $(\mathbf{A})$ all patients $(n=124)$ and $\mathbf{( B )}$ those without missing values in any of the four responder criteria $(n=58)$. 
TABLE 1 | Baseline information of the enrolled participants and the responder analysis based on exacerbation reduction and oral corticosteroid sparing.

\begin{tabular}{|c|c|c|c|c|c|c|c|}
\hline & \multicolumn{3}{|c|}{ Exacerbation reduction } & \multicolumn{3}{|c|}{ OCS sparing } & $\begin{array}{c}\text { Total } \\
(n=124)\end{array}$ \\
\hline Age (years) & & & 0.303 & & & 0.886 & \\
\hline Mean \pm SD & $61.4 \pm 15.9$ & $58.9 \pm 15.3$ & & $60.7 \pm 16.0$ & $61.6 \pm 14.7$ & & $60.8 \pm 15.7$ \\
\hline Median (Q1, Q3) & $64.0(50.8,74.0)$ & $57.0(48.5,68.3)$ & & $62.0(50.0,71.0)$ & $61.0(50.5,73.5)$ & & $62.0(50.0,71.0)$ \\
\hline BMI $\left(\mathbf{k g} / \mathrm{m}^{2}\right)^{\#}$ & & & $0.032^{*}$ & & & 0.792 & \\
\hline Mean \pm SD & $25.8 \pm 4.3$ & $27.6 \pm 4.5$ & & $26.2 \pm 4.5$ & $26.0 \pm 4.2$ & & $26.2 \pm 4.4$ \\
\hline Median (Q1, Q3) & $25.0(22.5,28.3)$ & $26.4(25.1,30.1)$ & & $25.3(23.3,29.6)$ & $25.6(22.6,27.5)$ & & $25.3(23.1,29.4)$ \\
\hline$<25$ & 47 (50.0\%) & $6(20.0 \%)$ & & $44(42.7 \%)$ & $9(42.9 \%)$ & & $53(42.7 \%)$ \\
\hline$\geqq 25,<30$ & $28(29.8 \%)$ & $15(50.0 \%)$ & & 35 (34.0\%) & $8(38.1 \%)$ & & $43(34.7 \%)$ \\
\hline$\geqq 30$ & 19 (20.2\%) & $9(30.0 \%)$ & & $24(23.3 \%)$ & $4(19.0 \%)$ & & $28(22.6 \%)$ \\
\hline$\geqq 10$ & 24 (25.5\%) & $11(36.7 \%)$ & & 29 (28.2\%) & $6(28.6 \%)$ & & 35 (28.2\%) \\
\hline Smoking history & & & 0.139 & & & 0.500 & \\
\hline Never smoker & 66 (70.2\%) & $16(53.3 \%)$ & & $68(66.0 \%)$ & $14(66.7 \%)$ & & $82(66.1 \%)$ \\
\hline Ex-smoker & 25 (26.6\%) & $11(36.7 \%)$ & & $29(28.2 \%)$ & 7 (33.3\%) & & 36 (29.0\%) \\
\hline Current smoker & $3(3.2 \%)$ & $3(10.0 \%)$ & & $6(5.8 \%)$ & $0(0.0 \%)$ & & $6(4.8 \%)$ \\
\hline \multicolumn{2}{|l|}{ Time for asthma history (years) } & & 0.407 & & & 0.934 & \\
\hline Mean \pm SD & $3.7 \pm 3.6$ & $4.0 \pm 3.2$ & & $3.8 \pm 3.6$ & $3.7 \pm 3.1$ & & $3.7 \pm 3.5$ \\
\hline Median (Q1, Q3) & $2.6(0.8,5.4)$ & $3.5(0.9,6.6)$ & & $2.9(0.8,5.7)$ & $2.0(0.9,6.1)$ & & $2.8(0.9,5.8)$ \\
\hline Total lgE (kU/L) & & & 0.764 & & & 0.813 & \\
\hline Mean \pm SD & $750.3 \pm 723.8$ & $722.3 \pm 757.4$ & & $725.2 \pm 693.8$ & $833.4 \pm 896.4$ & & $743.5 \pm 729.0$ \\
\hline$\geqq 300$ & $36(38.3 \%)$ & $10(33.3 \%)$ & & $31(30.1 \%)$ & $15(71.4 \%)$ & & $46(37.1 \%)$ \\
\hline Number of allergens tested & & & 1.000 & & & 0.245 & \\
\hline Mean \pm SD & $1.9 \pm 1.6$ & $1.9 \pm 1.4$ & & $2.0 \pm 1.5$ & $1.6 \pm 1.6$ & & $1.9 \pm 1.5$ \\
\hline Median (Q1, Q3) & $2.0(1.0,3.0)$ & $2.0(1.0,3.0)$ & & $2.0(1.0,3.0)$ & $1.0(0.0,3.0)$ & & $2.0(1.0,3.0)$ \\
\hline \multicolumn{2}{|c|}{ Initial Omalizumab dose (mg/month) } & & 0.488 & & & 0.362 & \\
\hline Mean \pm SD & $447.7 \pm 227.3$ & $478.5 \pm 232.4$ & & $465.8 \pm 239.1$ & $406.3 \pm 161.4$ & & $455.4 \pm 228.0$ \\
\hline Median (Q1, Q3) & $450.0(300.0,600.0)$ & $450.0(300.0,600.0)$ & & $450.0(300.0,600.0)$ & $450.0(300.0,525.0)$ & & $450.0(300.0,600.0)$ \\
\hline Inhaled medication & & & 1.000 & & & 0.475 & \\
\hline $\begin{array}{l}\text { Medium-dose ICS/LABA } \pm \\
\text { Tiotropium }\end{array}$ & 40 (42.6\%) & $13(43.3 \%)$ & & $46(44.7 \%)$ & $7(33.3 \%)$ & & $53(42.7 \%)$ \\
\hline $\begin{array}{l}\text { High-dose ICS/LABA } \pm \\
\text { Tiotropium }\end{array}$ & $54(57.4 \%)$ & $17(56.7 \%)$ & & 57 (55.3\%) & $14(66.7 \%)$ & & 71 (57.3\%) \\
\hline Oral medication & & & 0.296 & & & 0.235 & \\
\hline None & $7(9.4 \%)$ & $4(13.3 \%)$ & & $10(9.7 \%)$ & $1(4.8 \%)$ & & $11(8.9 \%)$ \\
\hline Montelukast alone & $53(56.4 \%)$ & $20(66.7 \%)$ & & $59(57.3 \%)$ & $14(66.7 \%)$ & & $73(58.9 \%)$ \\
\hline Methylxanthines alone & $12(12.8 \%)$ & $1(3.3 \%)$ & & $9(8.7 \%)$ & $4(19.0 \%)$ & & $13(10.5 \%)$ \\
\hline Montelukast + Methylxanthines & $22(23.4 \%)$ & $5(16.7 \%)$ & & $25(24.3 \%)$ & $2(9.5 \%)$ & & 27 (21.8\%) \\
\hline \multicolumn{2}{|c|}{ OCS maintenance dose (mg/month) } & & 0.351 & & & 0.173 & \\
\hline
\end{tabular}


TABLE 1 | Continued

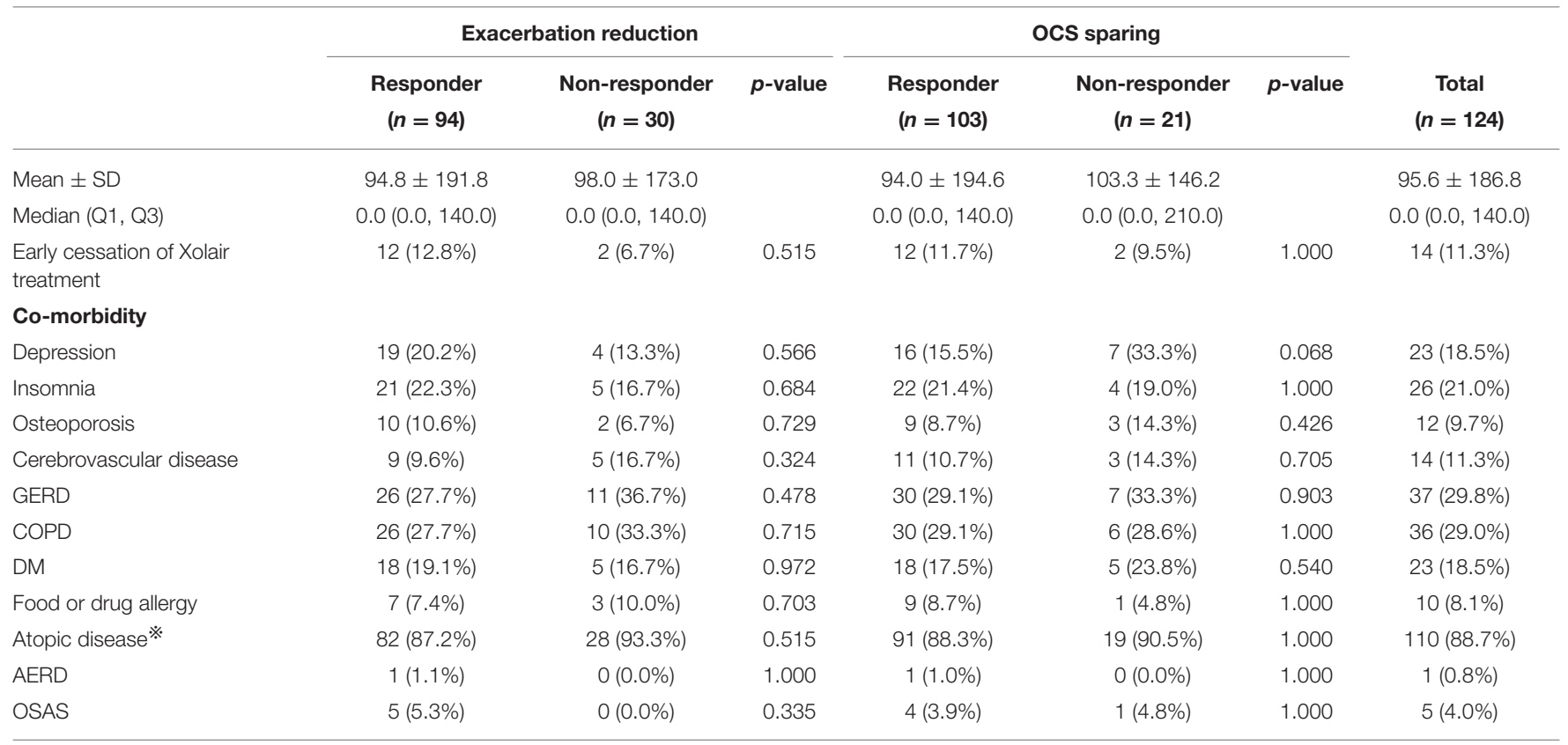

${ }^{*} p<0.05$.

\#Categorized based on the World Health Organization recommendations.

*Atopic disease included allergic dermatitis, allergic rhinitis, allergic conjunctivitis and food allergies.

$A E R D$, aspirin-exacerbated respiratory disease; BMI, body mass index; COPD, chronic obstructive pulmonary disease; DM, diabetes mellitus; GERD, gastro-esophageal reflux disease;

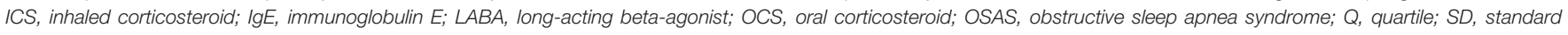
deviation; WBC, white blood count.

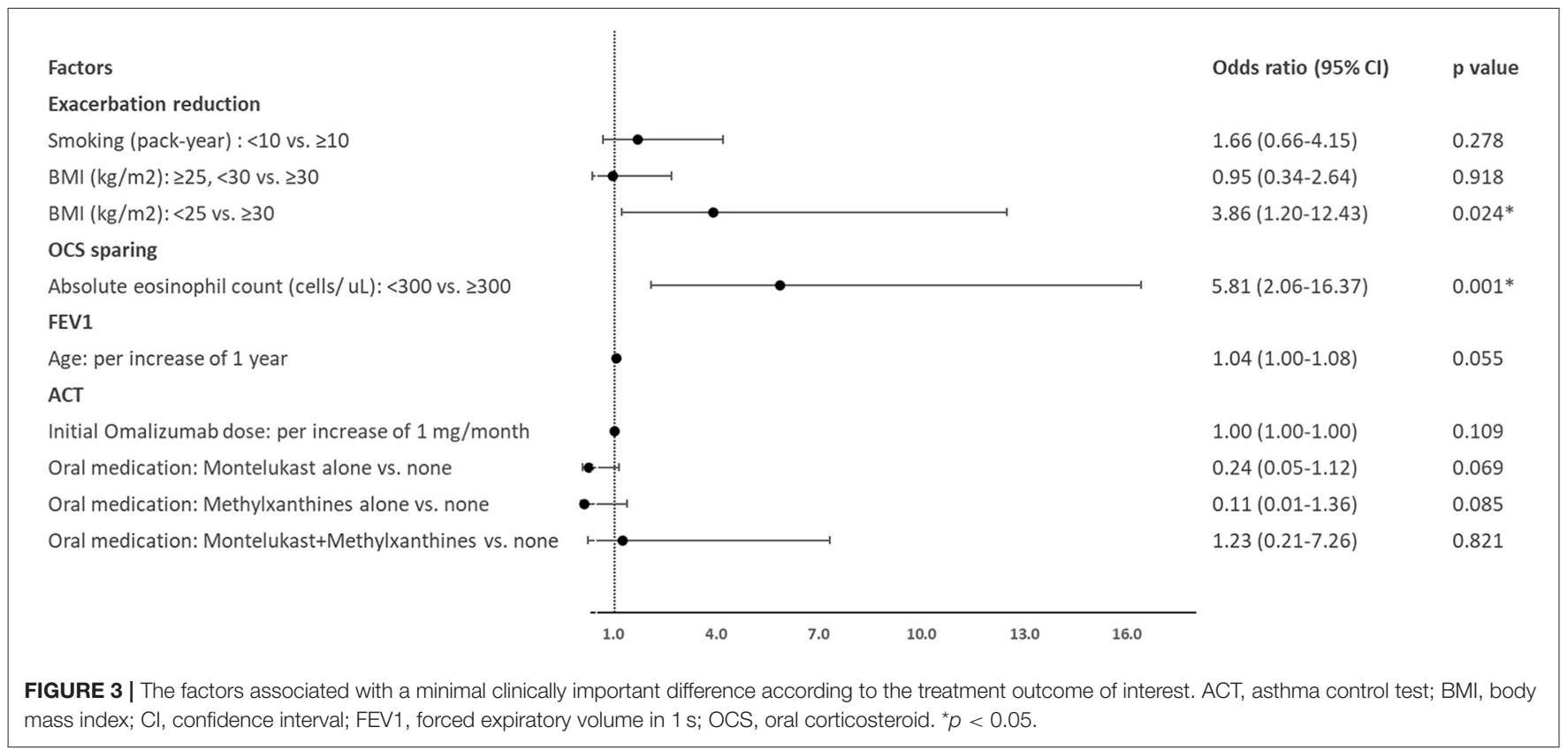

\section{Statistical Analysis}

Categorical variables were presented as frequency and percentage and compared using the chi-squared test between the responders and non-responders. Continuous variables were presented using mean and standard deviation, and median and inter-quartile range, and were compared using a paired sample $t$-test or Wilcoxon signed-rank-test according to the normality assumption between the study groups. Logistic regression models were used to analyze independent factors if they were significant in the univariate analysis for patients with binary results of 
TABLE 2 | The factors associated with the responder of lung function improvement.

\begin{tabular}{|c|c|c|c|c|}
\hline & \multicolumn{2}{|c|}{ FEV1 } & $p$-value & Total $(n=75)$ \\
\hline Mean \pm SD & $56.3 \pm 17.5$ & $63.8 \pm 13.2$ & & $61.8 \pm 14.7$ \\
\hline Median (Q1, Q3) & $54.5(42.5,71.5)$ & $65.0(55.0,70.0)$ & & $63.0(51.0,70.0)$ \\
\hline Male gender & $12(60.0 \%)$ & 27 (49.1\%) & 0.565 & 39 (52.0\%) \\
\hline Mean \pm SD & $27.2 \pm 5.3$ & $26.6 \pm 4.4$ & & $26.8 \pm 4.6$ \\
\hline Median (Q1, Q3) & $26.0(24.0,30.5)$ & $26.3(23.4,29.9)$ & & $26.3(23.5,30.2)$ \\
\hline Smoking (pack-year) & & & 0.875 & \\
\hline Mean \pm SD & $9.9 \pm 13.8$ & $10.4 \pm 18.4$ & & $10.3 \pm 17.2$ \\
\hline Median (Q1, Q3) & $0.0(0.0,20.0)$ & $0.0(0.0,20.0)$ & & $0.0(0.0,20.0)$ \\
\hline Smoking history & & & 0.545 & \\
\hline Time for asthma history (years) & & & 0.679 & \\
\hline Mean \pm SD & $3.2 \pm 2.8$ & $4.2 \pm 4.2$ & & $3.9 \pm 3.9$ \\
\hline Median (Q1, Q3) & $2.3(0.8,5.2)$ & $2.8(0.7,6.6)$ & & $2.8(0.8,6.5)$ \\
\hline Total IgE (kU/L) & & & 0.774 & \\
\hline Mean \pm SD & $614.2 \pm 443.3$ & $670.0 \pm 530.0$ & & $655.1 \pm 506.0$ \\
\hline Median (Q1, Q3) & $425.0(311.0,836.8)$ & $532.0(219.0,875.0)$ & & $510.0(289.0,860.0)$ \\
\hline WBC $\left(10^{9} / L\right)$ & & & 0.679 & \\
\hline Mean \pm SD & $9.0 \pm 2.9$ & $8.8 \pm 3.4$ & & $8.9 \pm 3.2$ \\
\hline Median (Q1, Q3) & $8.2(6.6,11.9)$ & $7.9(6.4,10.6)$ & & $7.9(6.5,10.8)$ \\
\hline Blood absolute eosinophil count (cells $/ \mu L$ ) & & & 0.375 & \\
\hline Median (Q1, Q3) & $450.0(300.0,600.0)$ & $412.5(300.0,600.0)$ & & $450.0(300.0,600.0)$ \\
\hline Inhaled medication & & & 0.522 & \\
\hline Medium-dose ICS/LABA \pm Tiotropium & $4(20.0 \%)$ & 17 (30.9\%) & & $21(28.0 \%)$ \\
\hline High-dose ICS/LABA \pm Tiotropium & $16(80.0 \%)$ & $38(69.1 \%)$ & & 54 (72.0\%) \\
\hline Oral medication & & & 0.833 & \\
\hline None & $2(10.0 \%)$ & $6(10.9 \%)$ & & $8(10.7 \%)$ \\
\hline Montelukast alone & $14(70.0 \%)$ & $33(60.0 \%)$ & & $47(62.7 \%)$ \\
\hline Methylxanthines alone & $2(10.0 \%)$ & $6(10.9 \%)$ & & $8(10.7 \%)$ \\
\hline Montelukast + Methylxanthines & $2(10.0 \%)$ & $10(18.2 \%)$ & & $12(16.0 \%)$ \\
\hline Initial OCS maintenance dose (mg/month) & & & 0.519 & \\
\hline Mean \pm SD & $87.5 \pm 191.2$ & $64.0 \pm 129.3$ & & $70.3 \pm 147.3$ \\
\hline Median (Q1, Q3) & $0.0(0.0,140.0)$ & $0.0(0.0,140.0)$ & & $0.0(0.0,140.0)$ \\
\hline Early cessation of Xolair treatment & $2(10.0 \%)$ & $5(9.1 \%)$ & 1.000 & $7(9.3 \%)$ \\
\hline \multicolumn{5}{|l|}{ Co-morbidity } \\
\hline Depression & 1 (5.0\%) & $8(14.5 \%)$ & 0.430 & $9(12.0 \%)$ \\
\hline Insomnia & $2(10.0 \%)$ & $12(21.8 \%)$ & 0.328 & $14(18.7 \%)$ \\
\hline Osteoporosis & 2 (10.0\%) & $5(9.1 \%)$ & 1.000 & 7 (9.3\%) \\
\hline Cerebrovascular disease & $1(5.0 \%)$ & $5(9.1 \%)$ & 1.000 & $6(8.0 \%)$ \\
\hline
\end{tabular}




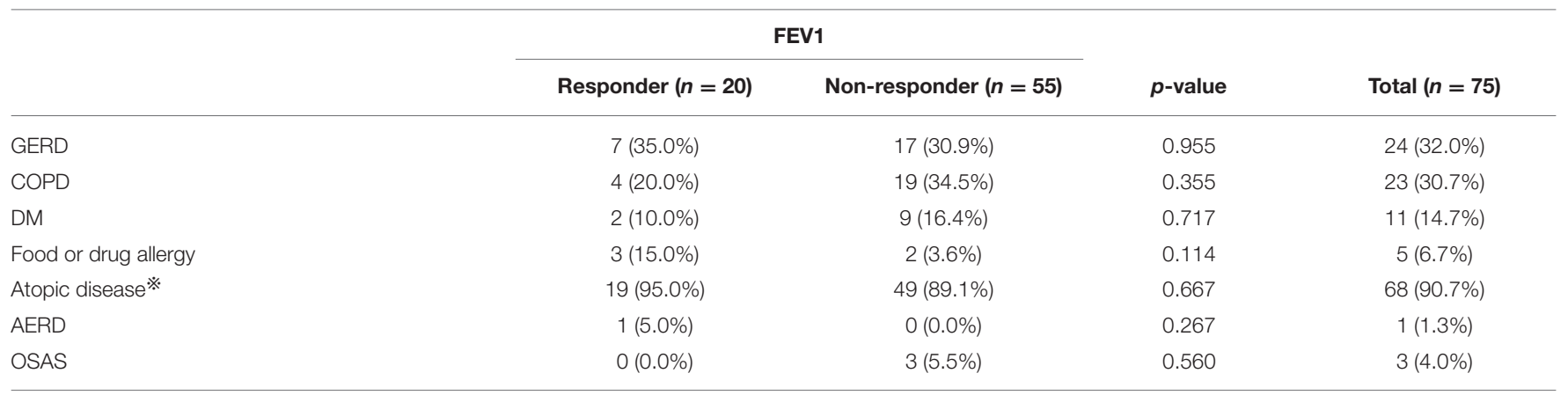

${ }^{*} p<0.05$.

*Atopic disease included allergic dermatitis, allergic rhinitis, allergic conjunctivitis and food allergies.

FEV1, forced expiratory volume in $1 \mathrm{~s}$; also see Table 1.

treatment outcomes of interest. A significant difference was defined as a two-sided $p$-value $<0.05$. The data of all patients were de-identified before analysis.

\section{RESULTS}

Figure 1 shows the patient enrolment flow chart. Of the 128 patients with SAA who applied for reimbursements for omalizumab during the study period, 124 received approval and were included in the final analysis. Among the 124 enrollees, 110 patients received omalizumab treatment for at least 10 months during the 1 -year follow-up period while 14 subjects received only 4 months of omalizumab because of the administrative issue from the Taiwan NHI (8). Only 75 and 85 patients had binary results of lung function measurements and ACT scores, respectively, at enrollment and the end of the 1-year follow-up period for further investigation. Of the 124 patients, 94 (75.8\%), 103 (83.1\%), 20 (26.7\%), and 53 $(62.4 \%)$ were identified as responders according to a reduction in exacerbations, OCS sparing, and improvements in lung function and asthma control, respectively.

Nearly all of the patients met one or more of the responder criteria, while only $4.8 \%$ of the patients with complete data and $10.3 \%$ of the those with missing values met all four responder criteria, respectively (Figure 2).

The mean age of the 124 patients was $60.8 \pm 15.7$ years (Table 1). More than a quarter of the patients had substantial smoking exposure, defined as $\geqq 10$ pack-years of cigarette smoking in their lifetime $(35 / 124,28.2 \%)$, while less than half were normal weight, de-fined as a body mass index $<25 \mathrm{~kg} / \mathrm{m}^{2}$ $(53 / 124,42.7 \%)$. Furthermore, of the 124 patients, 46 (37.1\%) had blood eosinophilia of $\geqq 300$ cells/ $\mu \mathrm{L}$ (Table $\mathbf{1}$ ).

Compared to the non-responders regarding reduction in exacerbations, the responders had a significantly higher and lower proportion of normal weight and substantial smoking exposure, respectively (Table 1), while the former was independently associated with an MCID in a reduction in exacerbations (Figure 3).

Responders with OCS sparing had a lower blood eosinophil level expressed by absolute count (cells/ $\mu \mathrm{L}$ ) compared to the non-responders (Table 1). The logistic regression analysis showed that $<300$ cells $/ \mu \mathrm{L}$ of circulating eosinophils was a significant predictor of an MCID in the sparing of OCS to control asthma (Figure 3).

The patients who exhibited an MCID in FEV1 improvement following omalizumab treatment were younger than those who did not (Table 2), although a younger age was not independently predictive of this treatment outcome (Figure 3).

The responders with an improvement in ACT following omalizumab treatment were associated with a higher initial dose of omalizumab and more use of either montelukast alone or methylxanthines alone to control asthma (Table 3). None of these characteristics could independently predict an MCID in an improvement in ACT score (Figure 3).

\section{DISCUSSION}

In this study of 124 adult patients with SAA, 75.8, 83.1, 26.7, and $62.4 \%$ were considered to be responders following omalizumab treatment according to a reduction in exacerbations, OCS sparing, and improvements in lung function and asthma control, respectively. The responders with a reduction in exacerbations were characterized by normal weight and less smoking exposure; OCS sparing by a lower blood eosinophil level; lung function improvement by a younger age; and asthma control improvement by a higher initial dose of omalizumab and more use of either montelukast alone or methylxanthines alone to control asthma. In addition, normal weight was a significant predictor of an MCID in a reduction in exacerbations following omalizumab treatment; a circulatory eosinophil level of $<300$ cells $/ \mu \mathrm{L}$ in OCS sparing; and none in improvements in lung function or asthma control.

Our results showed that normal weight and less smoking exposure were associated with an MCID in a reduction in the annual number of exacerbations, and the former was an independent predictor. In contrast to our study, Casale et al. enrolled a relatively young population (a mean age of 47.3 years) with allergic asthma who were candidates for omalizumab treatment, and found that those with an increased number of exacerbations in the year prior to the study as well as female 
TABLE 3 | The responder analysis for the asthma control improvement.

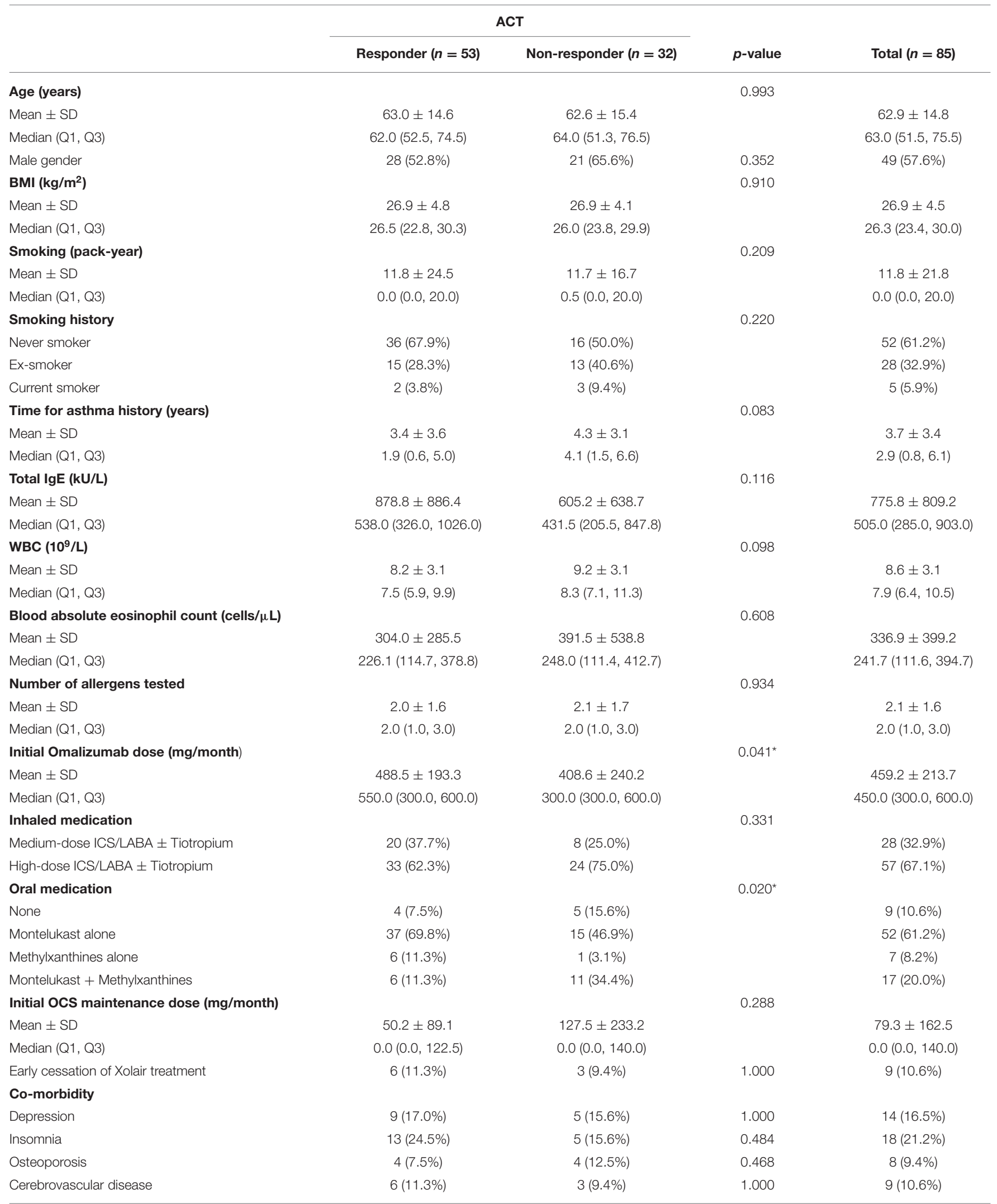




\begin{tabular}{|c|c|c|c|c|}
\hline & \multicolumn{2}{|c|}{ ACT } & $p$-value & Total $(n=85)$ \\
\hline COPD & $14(26.4 \%)$ & 15 (46.9\%) & 0.091 & 29 (34.1\%) \\
\hline $\mathrm{DM}$ & $12(22.6 \%)$ & $3(9.4 \%)$ & 0.207 & 15 (17.6\%) \\
\hline Food or drug allergy & 5 (9.4\%) & 2 (6.3\%) & 0.706 & 7 (8.2\%) \\
\hline AERD & $0(0.0 \%)$ & $0(0.0 \%)$ & NA & $0(0.0 \%)$ \\
\hline OSAS & $4(7.5 \%)$ & 0 (0.0\%) & 0.292 & 4 (4.7\%) \\
\hline
\end{tabular}

${ }^{*} p<0.05$.

*Atopic disease included allergic dermatitis, allergic rhinitis, allergic conjunctivitis and food allergies.

$A C T$, asthma control test; also see Table 1

patients were more likely to be responders with a reduction in exacerbations defined using a similar criteria to our study $(5,17)$. The inconsistencies in the features between our study and Casale et al.'s may mainly be due to the disparity in age of the study populations (a mean age of 60.8 vs. 47.3 years) (5). In addition, previous studies have reported that obesity, co-morbidities and the presence of nasal polyps were risk factors for exacerbations, while more clinically severe asthma (as evaluated by emergency visits and hospitalizations for asthma in the previous year, FEV1 \% predicted $<65$ vs. $\geq 65$, inhaled beclomethasone dipropionate dose $<600$ vs. $\geq 600 \mu$ g per day, and long-acting beta-agonist use vs. non-use) and high Th2driven inflammatory biomarkers (including FeNO, peripheral blood eosinophils, and serum periostin and total IgE) were associated with a greater reduction in exacerbations following treatment with omalizumab $(4,6,18,19)$.

We also found that a peripheral blood eosinophil level of $<300$ cells $/ \mu \mathrm{L}$ could predict responders with OCS sparing. Hanania et al. reported that a high level of FeNO could predict less albuterol use (4). In contrast, Sposato et al. reported that obesity was independently associated with the excessive use of shortacting beta-agonists and increased dose of medications to control asthma, and that nasal polyps were associated with the use of a higher inhaler corticosteroid dose (18).

Further, our study showed that the responders with lung function improvement were younger compared to the nonresponders, and that the patients with a higher initial omalizumab dose and more use of oral medications to control asthma were associated with an MCID in asthma control. In contrast, Casale et al. reported that patients with uncontrolled asthma and receiving asthma medications in addition to inhaled corticosteroids (ICS)/long-acting beta-agonists were more likely to be associated with an MCID in lung function, defined as a rise in FEV $1 \geqq 120 \mathrm{ml}$ from baseline, while those with higher baseline circulatory eosinophil levels were more likely to be responders with asthma control according to a definition similar to ours (5). Again, the discrepancy in the characteristics between these two studies may be explained by the difference in age of the study population (a mean age of 60.8 vs. 47.3 years) and in the definition of a response in lung function (an FEV1 improvement of $\geqq 230$ vs. $\geqq 120 \mathrm{ml})(5,17)$. Conversely, Sposato et al. reported that patients had a reduced response following omalizumab treatment in both lung function and asthma control if they were older or obese, and had co-morbidities, particularly chronic heart disease (18). Taken together, these findings suggest that the SAA patients who had an MCID in the treatment outcomes of interest following omalizumab treatment had a predictable clinical behavior, although the discrepancies in age and in the definition of a responder led to differences in the predictors.

Obese patients with asthma have an increased risk of severe disease, which may arise from many factors, such as changes in airway anatomy, adipokines, glucose-insulin metabolism, oxidative stress, inflammation, and genetic and epigenetic variants (20). Furthermore, similar to our finding that the SAA patients with normal weight were more likely to be responders with a reduction in exacerbations, previous reports have shown that obesity may reduce ICS response and negatively influence the beneficial effect of omalizumab in terms of asthma control in patients with asthma $(1,21)$. These findings show that obesity has a great impact on the severity and prognosis of asthma, and that obesity is a particular phenotype of asthma.

The predictive value of total IgE and blood eosinophil levels with regards to the therapeutic benefit of omalizumab in patients with allergic asthma has yet to be shown in previous studies, although we found that a blood eosinophil level of $<300$ cells $/ \mu \mathrm{L}$ was predictive of an MCID in OCS sparing in the SAA patients $(7,19,22)$. This disparity could be explained by the use of different treatment outcomes for analysis between the present study and other studies (OCS sparing vs. reduction in exacerbations).

Similar to the response rates of 75.8 and $62.4 \%$ for a reduction in exacerbations and improvement in asthma control, respectively, Casale et al. reported response rates of 77.8 and $64.7 \%$ (5). However, we found a lower response rate of $26.7 \%$ for an improvement in lung function defined as an increase in FEV1 $\geqq 230 \mathrm{ml}$ between enrollment and the end of the study compared to $35.9 \%$ defined as a $\geqq 120 \mathrm{ml}$ improvement in FEV1 between the end and start of the study reported by Casale et al. $(5,13,23)$. As expected, the definition of a responder affected the 
difference in response rate of treatment outcomes of interest in the asthmatic patients.

The main strength of the current study is that the diagnosis of SAA was made according to the GINA recommendations by clinicians who were actively involved in the management of asthma, while the initiation of omalizumab treatment in all patients was suggested by both the physician in charge and the Taiwan NHI committee for SAA (1), This ensured a valid study population of patients with SAA and reached a strong consensus on whether or not omalizumab should be an add-on therapy for SAA patients, although 14 of the 124 participants discontinued omalizumab treatment after 4 months because of administrative issues with the Taiwan NHI. This may compensate for the limitations of this study, which include the incomplete binary results of lung function tests and ACT data, older age of the participants, and the small number of cases. Due to these limitations, our results should be interpreted with caution, and they may not be generalizable to a younger population.

\section{CONCLUSIONS}

It is extremely important to take clinical features into consideration when managing patients with asthma at different GINA steps, particularly at GINA step 5, because several inflammatory biomarkers used to predict a good therapeutic response to the biologic treatment, such as FeNO and periostin, are not always available in all hospitals or areas/countries. We identified several clinical characteristics associated with an MCID in terms of a reduction in exacerbations, OCS sparing, and improvements in lung function and asthma control in adult patients with SAA. This information could help when selecting patients who may benefit more from omalizumab treatment to manage asthma. Future well-designed studies including more subjects and more potential and easily-obtained inflammatory biomarkers are warranted to more accurately predict an MCID following omalizumab treatment.

\section{REFERENCES}

1. Global Strategy for Asthma Management and Prevention. (2021). Available online at: https://ginasthma.org/gina-reports/ (accessed July 01, 2021).

2. Hekking PW, Wener RR, Amelink M, Zwinderman AH, Bouvy ML, Bel EH. The prevalence of severe refractory asthma. J Allergy Clin Immunol. (2015) 135:896-902. doi: 10.1016/j.jaci.2014.08.042

3. Kelly HW. Rationale for the major changes in the pharmacotherapy section of the National Asthma Education and Prevention Program guidelines. $J$ Allergy Clin Immunol. (2007) 120:989-94; quiz 995-6. doi: 10.1016/j.jaci.2007. 01.035

4. Hanania NA, Wenzel S, Rosén K, Hsieh HJ, Mosesova S, Choy DF, et al. Exploring the effects of omalizumab in allergic asthma: an analysis of biomarkers in the EXTRA study. Am J Respir Crit Care Med. (2013) 187:80411. doi: 10.1164/rccm.201208-1414OC

5. Casale TB, Luskin AT, Busse W, Zeiger RS, Trzaskoma B, Yang M, et al. Omalizumab effectiveness by biomarker status in patients with asthma: evidence from PROSPERO, a prospective real-world study. J Allergy Clin Immunol Pract. (2019) 7:156-64.e1. doi: 10.1016/j.jaip.2018.04.043

\section{DATA AVAILABILITY STATEMENT}

The original contributions presented in the study are included in the article/supplementary material, further inquiries can be directed to the corresponding author.

\section{ETHICS STATEMENT}

The studies involving human participants were reviewed and approved by the Institutional Review Board and Ethics Committee of Taichung Veterans General Hospital (Approval No. CE19015B). Written informed consent for participation was not required for this study in accordance with the national legislation and the institutional requirements.

\section{AUTHOR CONTRIBUTIONS}

W-CH: conceptualization, methodology, formal analysis, investigation, data curation, writing-original draft preparation, visualization, project administration, and agreement on the published version of the manuscript. P-KF, M-CC, C-SC, W-NH, K-LL, J-LW, W-TH, Y-DW, C-WH, and M-FW: methodology, formal analysis, investigation, data curation, writing-review and editing, visualization, and agreement on the published version of the manuscript. Y-HC and J-YH: conceptualization, methodology, validation, formal analysis, investigation, data curation, writing - review and editing, visualization, supervision, and agreement on the published version of the manuscript. All authors contributed to the article and approved the submitted version.

\section{ACKNOWLEDGMENTS}

The authors would like to thank Dr. Gwan-Han Shen, who supervised Laboratory No. 114 at Taichung Veterans General Hospital and passed away in 2014. We hold you dear in our memory.

6. Casale TB, Chipps BE, Rosén K, Trzaskoma B, Haselkorn T, Omachi TA, et al. Response to omalizumab using patient enrichment criteria from trials of novel biologics in asthma. Allergy. (2018) 73:490-7. doi: 10.1111/all. 13302

7. Humbert M, Taillé C, Mala L, Le Gros V, Just J, Molimard M, et al. Omalizumab effectiveness in patients with severe allergic asthma according to blood eosinophil count: the STELLAIR study. Eur Respir J. (2018) 51:1702523. doi: 10.1183/13993003.02523-2017

8. Huang WC, Fu PK, Chan MC, Chin CS, Huang WN, Lai KL, et al. The long-term effectiveness of omalizumab in adult patients with severe allergic asthma: continuous treatment versus boosting treatment. J Clin Med. (2021) 10:707. doi: 10.3390/jcm 10040707

9. Lanier B, Bridges T, Kulus M, Taylor AF, Berhane I, Vidaurre CF. Omalizumab for the treatment of exacerbations in children with inadequately controlled allergic (IgE-mediated) asthma. J Allergy Clin Immunol. (2009) 124:12106. doi: 10.1016/j.jaci.2009.09.021

10. Milgrom H, Berger W, Nayak A, Gupta N, Pollard S, McAlary M, et al. Treatment of childhood asthma with anti-immunoglobulin E antibody (omalizumab). Pediatrics. (2001) 108:E36. doi: 10.1542/peds.108.2.e36 
11. Jaeschke R, Singer J, Guyatt GH. Measurement of health status. Ascertaining the minimal clinically important difference. Control Clin Trials. (1989) 10:407-15. doi: 10.1016/0197-2456(89)90005-6

12. Bonini M, Di Paolo M, Bagnasco D, Baiardini I, Braido F, Caminati $\mathrm{M}$, et al. Minimal clinically important difference for asthma endpoints: an expert consensus report. Eur Respir Rev. (2020) 29:190137. doi: 10.1183/16000617.0137-2019

13. Santanello NC, Zhang J, Seidenberg B, Reiss TF, Barber BL. What are minimal important changes for asthma measures in a clinical trial? Eur Respir J. (1999) 14:23-7. doi: 10.1034/j.1399-3003.1999.14a06.x

14. Schatz M, Kosinski M, Yarlas AS, Hanlon J, Watson ME, Jhingran P. The minimally important difference of the Asthma Control Test. J Allergy Clin Immunol. (2009) 124:719-23.e1. doi: 10.1016/j.jaci.2009. 06.053

15. Castro M, Wenzel SE, Bleecker ER, Pizzichini E, Kuna P, Busse WW, et al. Benralizumab, an anti-interleukin 5 receptor $\alpha$ monoclonal antibody, versus placebo for uncontrolled eosinophilic asthma: a phase $2 \mathrm{~b}$ randomised dose-ranging study. Lancet Respir Med. (2014) 2:87990. doi: 10.1016/S2213-2600(14)70201-2

16. Castro M, Zangrilli J, Wechsler ME, Bateman ED, Brusselle GG, Bardin P, et al. Reslizumab for inadequately controlled asthma with elevated blood eosinophil counts: results from two multicentre, parallel, double-blind, randomised, placebo-controlled, phase 3 trials. Lancet Respir Med. (2015) 3:355-66. doi: 10.1016/S2213-2600(15)00042-9

17. Chipps BE, Zeiger RS, Luskin AT, Busse WW, Trzaskoma BL, Antonova EN, et al. Baseline asthma burden, comorbidities, and biomarkers in omalizumabtreated patients in PROSPERO. Ann Allergy Asthma Immunol. (2017) 119:524-32.e2. doi: 10.1016/j.anai.2017.09.056

18. Sposato B, Scalese M, Milanese M, Masieri S, Cavaliere C, Latorre $\mathrm{M}$, et al. Factors reducing omalizumab response in severe asthma. Eur J Intern Med. (2018) 52:78-85. doi: 10.1016/j.ejim.2018. 01.026
19. Bousquet J, Rabe K, Humbert M, Chung KF, Berger W, Fox H, et al. Predicting and evaluating response to omalizumab in patients with severe allergic asthma. Respir Med. (2007) 101:1483-92. doi: 10.1016/j.rmed.2007.01.011

20. Sutherland ER. Linking obesity and asthma. Ann N Y Acad Sci. (2014) 1311:31-41. doi: 10.1111/nyas.12357

21. Gu C, Upchurch K, Mamaril-Davis J, Wiest M, Lanier B, Millard M, et al. Obesity influences the outcomes of anti-IgE (omalizumab) therapy of asthma. Clin Exp Allergy. (2020) 50:1196-9. doi: 10.1111/cea.13696

22. Busse WW. Are peripheral blood eosinophil counts a guideline for omalizumab treat-ment? STELLAIR says no! Eur Respir J. (2018) 51:1800730. doi: 10.1183/13993003.00730-2018

23. Donohue JF. Minimal clinically important differences in COPD lung function. COPD. (2005) 2:111-24. doi: 10.1081/COPD-200053377

Conflict of Interest: The authors declare that the research was conducted in the absence of any commercial or financial relationships that could be construed as a potential conflict of interest.

Publisher's Note: All claims expressed in this article are solely those of the authors and do not necessarily represent those of their affiliated organizations, or those of the publisher, the editors and the reviewers. Any product that may be evaluated in this article, or claim that may be made by its manufacturer, is not guaranteed or endorsed by the publisher.

Copyright (c) 2022 Huang, Fu, Chan, Chin, Huang, Lai, Wang, Hung, Wu, Hsieh, Wu, Chen and Hsu. This is an open-access article distributed under the terms of the Creative Commons Attribution License (CC BY). The use, distribution or reproduction in other forums is permitted, provided the original author(s) and the copyright owner(s) are credited and that the original publication in this journal is cited, in accordance with accepted academic practice. No use, distribution or reproduction is permitted which does not comply with these terms. 